\title{
ANNOTATIONS
}

\section{Needle Biopsy in the Diagnosis of Malignant Deposits in Bone}

Aspirating tumour tissue through a needle is a simple procedure and is a method of biopsy that may be extremely useful in certain cases of diagnostic difficulty. Two indications for its use are (I) where a probable bony metastasis is found in a patient who manifests no evidence of a primary growth and (2) where a secondary deposit is suspected and proof of its presence would alter the proposed treatment of the primary disease. Two of our cases may be quoted as examples. One patient with a hypernephroma of the kidney was found on X-ray to have a lesion of the body of the tenth dorsal vertebra that might have been the result of an old injury. Needle biopsy of the affected bone gave evidence of secondary carcinoma. Post mortem later confirmed these findings. Another had been admitted to a neurological hospital with symptoms of an intracranial aneurysm, but percutaneous angiography was normal. Mild backache led to an X-ray of the lumbar spine and this showed an osteolytic lesion of the body of L3. Needle biopsy produced cells probably metastasising from an 'oat-cell ' carcinoma of the lung. Although at that time a chest X-ray was clear, a carcinoma of the bronchus was later found to be, in fact, present, together with deposits in the skull and other bones.

Coley et al. (193I) and Ellis (1947) have given comprehensive accounts of the needle biopsy of many different tissues. Ellis included a large and successful series carried out with few complications. His indications for this procedure included (I) when a tumour lies deep to normal skin or other tissues, (2) when an operation would otherwise be necessary and yet such an operation would not be curative, and ( 3 ) when the usual methods of biopsy are contraindicated. Stewatt (1933) found no untoward reactions in 2,500 cases.
Using the simplest of techniques, we have found this investigation to be worthwhile. Local anaesthesia has been preferred, but a general anaesthetic is permissible. A long needle of No. 16 gauge may be used, but we prefer a $3 \frac{3}{4}$-in. 16-gauge Barker or Bier spinal needle when it is long enough, as it has a well-fitting stilette. The skin is punctured with a scalpel in order to prevent epithelial contamination and blockage of the needle, which is then passed in the appropriate direction. This is easier than one expects, as the lesion is usually larger than the radiological appearances would suggest. Should there be difficulty the insertion may be made under $\mathrm{X}$-ray control. Often there is a sensation of softness; when this is felt the lesion has been reached and strong suction with a $20-\operatorname{ce}$ syringe should be applied and at the same time the needle is advanced and rotated. Severat on specimens should be obtained and the syringe repeatedly rinsed in saline; after the needle has been withdrawn it is cleared with a wire. The fragments are fixed in formalin, embedded in wax and sectioned in the ordinary way. Semi-solid specimens may be prepared in the form of a smear. Staining techniques are given in the papers quoted.

We have successfully aspirated tumour cello from all three regions of the spine and also from the vertebral ends of ribs. In every case we have been grateful for the information, yet in such places any other method of biopsy is rarely indicated.

Even though the indications may be less than its champions claim, we have found this comparatively trivial procedure to be of great assistance and recommend its use in all cases of doubtful bone secondaries. Seventy per cent. of such $\mathcal{O}$ attempts will be rewarding.

\section{L. B. FARLEY, F.R.C.S.}

\section{BIBLIOGRAPHY}

COLEY, B. L., SHARP, G. S., and ELLIS, E. B. (193I), Amer. 7. Surg, 13, 215.

ELLIS, FRANK (1947), Brit. F. Surg., 34, 240.

STEWART, F. W. (1933), Amer. F. Path., 9, 801. 